\title{
Detecting Consistent Common Lines in Cryo-EM by Voting
}

\author{
Amit Singer ${ }^{\mathrm{a}}$, Ronald R. Coifman ${ }^{\mathrm{b}}$, Fred J. Sigworth ${ }^{\mathrm{c}}$, David W. Chester ${ }^{\mathrm{c}}$, \\ Yoel Shkolnisky ${ }^{\mathrm{b}}$ \\ ${ }^{a}$ Department of Mathematics and PACM, Princeton University, Fine Hall, Washington Road, \\ Princeton NJ 08544-1000 USA. \\ ${ }^{b}$ Department of Mathematics, Program in Applied Mathematics, Yale University, 10 Hillhouse Ave. \\ PO Box 208283, New Haven, CT 06520-8283 USA. \\ ${ }^{c}$ Department of Cellular and Molecular Physiology, Yale University School of Medicine, 333 Cedar \\ Street, New Haven, CT 06520 USA.
}

\begin{abstract}
The cryo-electron microscopy (EM) reconstruction problem is to find the three-dimensional structure of a macromolecule given its two-dimensional noisy projection images at unknown random directions. Ab initio estimates of the 3D structure are often obtained by the "Angular Reconstitution" method, in which a coordinate system is established from three projections, and the orientation of the particle giving rise to each image is deduced from common lines among the images. However, a reliable detection of common lines is difficult due to the low signal-to-noise ratio of the images. In this paper we describe a global self-correcting voting procedure in which all projection images participate to decide the identity of the consistent common lines. The algorithm determines which common line pairs were detected correctly and which are spurious. We show that the voting procedure succeeds at relatively low detection rates and that its performance improves as the number of projection images increases. We demonstrate the algorithm for both simulative and experimental images of the E. coli ribosome.
\end{abstract}

\section{Introduction}

"Three-dimensional electron microscopy" [Frank, 2006] is the name commonly given to methods in which the 3D structures of macromolecular complexes are obtained from sets of images taken in an electron microscope. The most widespread and general of these methods is single-particle reconstruction (SPR). In SPR the 3D structure is determined from images of randomly oriented and positioned, identical macromolecular "particles", typically complexes $500 \mathrm{kDa}$ or larger in size. The SPR method has been applied to images of negatively stained specimens, and to images obtained from frozenhydrated, unstained specimens [Wang and Sigworth, 2006]. In the latter technique,

Email addresses: amits@math.princeton.edu (Amit Singer), coifman-ronald@yale.edu (Ronald R. Coifman), fred.sigworth@yale.edu (Fred J. Sigworth), david.chester@yale.edu (David W. Chester), yoel.shkolnisky@yale.edu (Yoel Shkolnisky) 
called cryo-EM, samples are freeze quenched in vitreous ice and maintained at a holding temperature around $-180^{\circ}$ throughout image acquisition.

SPR from cryo-EM images is of particular interest because it promises to be an entirely general technique. It does not require crystallization or other special preparation of the complexes to be imaged, and is beginning to reach sufficient resolution $(\sim 0.4 \mathrm{~nm})$ to allow the polypeptide chain to be traced and residues identified in protein molecules [Henderson, 2004; Ludtke, S. J. et al., 2008; Zhang, X. et al., 2008]. Even at the resolutions of 0.6-0.9 nm, many important features of protein molecules can be determined [Chiu et al., 2005].

Much progress has been made in algorithms that, given a starting 3D structure, are able to refine that structure on the basis of a set of negative-stain or cryo-EM images, which are taken to be projections of the $3 \mathrm{D}$ object. Data sets typically range from $10^{4}$ to $10^{5}$ particle images, and refinements require tens to thousands of CPU-hours. As the starting point for the refinement process, however, some sort of ab initio estimate of the 3D structure must be made. Present algorithms are based on the "Angular Reconstitution" method of van Heel [van Heel, 1987] in which a coordinate system is established from three projections, and the orientation of the particle giving rise to each image is deduced from common lines among the images. However, conventional SPR algorithms fail with particles that are too small, with images that are too noisy, or at resolutions where the signal-to-noise ratio becomes too small.

The common lines between three projections uniquely determine their relative orientations up to handedness (chirality). This is the basis of the angular reconstitution method of Van Heel [van Heel, 1987], which was also developed independently by Vainshtein and Goncharov [Vainshtein, B. and Goncharov, A., 1986]. Farrow and Ottensmeyer [Farrow, M. and Ottensmeyer, P., 1992] used quaternions to obtain the relative orientation of a new projection in a least squares sense. The main problem with such sequential approaches is that they are sensitive to false detection of common lines which leads to the accumulation of errors. Penczek et al. [Penczek et al., 1996] tried to obtain the rotations corresponding to all projections simultaneously by minimizing a global energy functional. Unfortunately, minimization of the energy functional requires a brute force search in a huge parametric space of all possible orientations for all projections. Mallick et al. [Mallick, S. P. et al., 2006] suggested an alternative Bayesian approach, in which the common line between a pair of projections can be inferred from their common lines with different projection triplets. The problem with this particular approach is that it requires too many (at least seven) common lines to be correctly identified simultaneously. Therefore, it is not suitable in cases where the true detection rate of common lines is small.

In this paper we introduce a Bayesian approach that requires only a small fraction of the common lines to be correctly identified. Without knowing which common lines are correct and which are false, our method is able to separate the good from the bad by boosting the good information and averaging out the bad. The paper is organized in the following way. In Section 2 we revisit the Fourier projection-slice theorem and the concept of common lines. In Section 3 we describe the global voting procedure and the 
way it distinguishes the good common line pairs from the bad pairs. During the voting procedure many "votes" are being disqualified, as explained in Section 4. Section 5 details the results of numerous numerical experiments using simulative artificial images and real electron microscope images of the E. coli ribosome. Using the voting procedure we were able to recover directly the $3 \mathrm{D}$ structure of the ribosome from 750,1500 , and 3000 class averages, generated from a data set of 27,121 projections. Simulation results provide quantitative measures for the ability of the voting procedure to find consistent common lines from low SNR images, for which many of the common lines are incorrect. Finally, Section 6 is a summary and discussion.

\section{Fourier projection-slice theorem and common lines}

The cryo-EM reconstruction problem is to find the three-dimensional structure of a molecule given a finite set of its two-dimensional projection images at unknown random directions. The intensity of pixels in a given projection image corresponds to line integrals of the electric potential $\phi(x, y, z)$ induced by the molecule along the path of the imaging electrons (Radon transform). The highly intense electron beam destroys the molecule and it is therefore impractical to take projection images of the same molecule at known different directions, as in the case of classical computerized tomography. In other words, a single molecule can be imaged only once. All molecules are assumed to have the exact same structure; they differ only by their spatial orientation. Thus, every image is a projection of the same molecule but at an unknown random orientation. The cryo-EM problem is thus stated as follows: find $\phi(x, y, z)$ given a collection of projection images.

One of the cornerstones of tomography is the Fourier projection-slice theorem, which states that the two-dimensional Fourier transform of a projection image is a planar slice (perpendicular to the beaming direction) of the three-dimensional Fourier transform of the molecule (see, e.g., [Natterer, 2001, p. 11]). The geometry induced by the Fourier projection-slice theorem is illustrated in Figure 1. Any two slices share a common line, i.e., the intersection line of the two planes. Every radial line in the two-dimensional Fourier transform of a projection image is also a radial line in the three-dimensional

Fourier transform of the molecule (see for example $\boldsymbol{\Lambda}_{k_{1}, l_{1}}$ in Figure 1). Moreover, there is a 1-to-1 correspondence between each radial line in the three-dimensional Fourier space and its direction vector in $\mathbb{R}^{3}$ (see for example $\boldsymbol{\Lambda}_{k_{1}, l_{1}}$ and $\boldsymbol{\beta}_{k_{1}, l_{1}}$ in Figure 1). The set of all direction vectors (unit vectors in $\mathbb{R}^{3}$ ) is known as the unit sphere. The radial lines of a single projection image correspond to a great geodesic circle on the unit sphere. The common line property can now be restated as follows: any two different geodesic circles over the unit sphere intersect at exactly two antipodal points. This is demonstrated at the bottom right part of Figure 1.

Common lines between pairs of projections are usually found using correlation. Given a data set of $N$ projection images $P_{1}(x, y), \ldots, P_{N}(x, y)$, one first computes the 


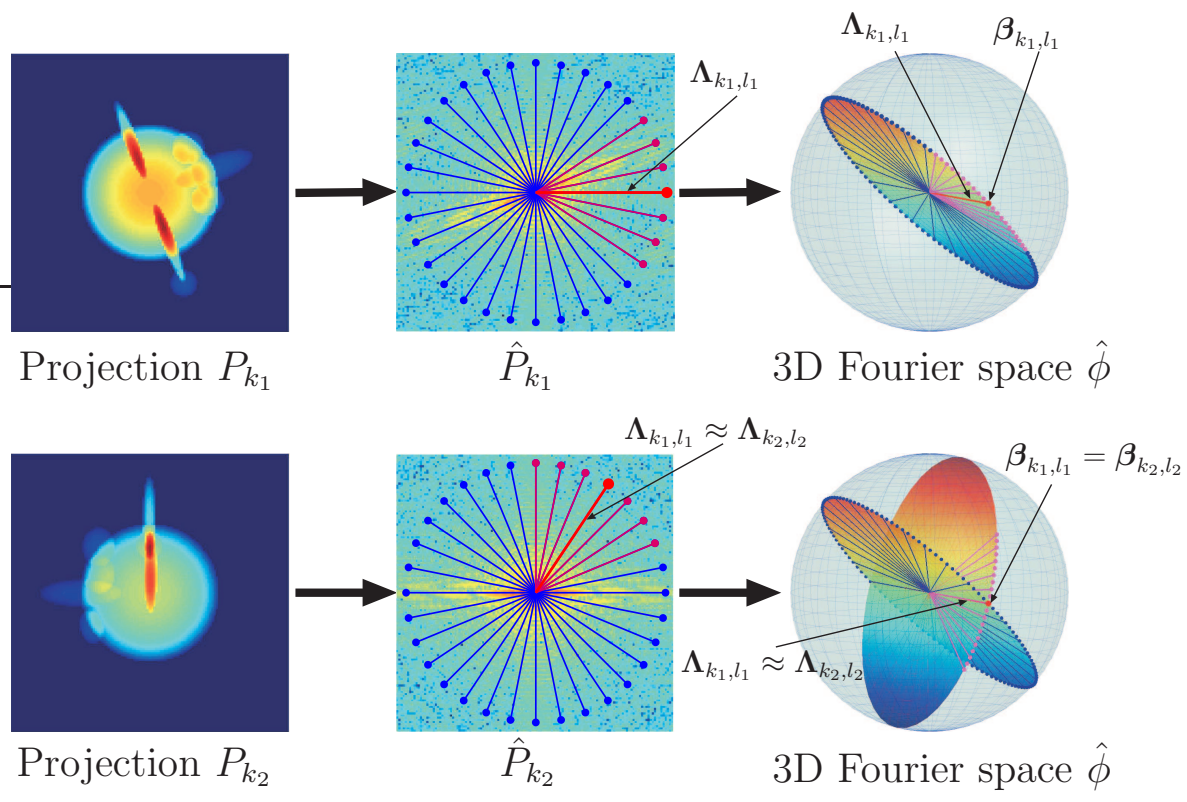

Figure 1: Fourier projection-slice theorem and its induced geometry. The Fourier transform of each projection $\hat{P}_{k}$ corresponds to a planar slice through the three-dimensional Fourier transform $\hat{\phi}$ of the molecule. The Fourier transforms of any two projections $\hat{P}_{k_{1}}$ and $\hat{P}_{k_{2}}$ share a common line $\left(\boldsymbol{\Lambda}_{k_{1}, l_{1}}\right.$ and $\left.\boldsymbol{\Lambda}_{k_{2}, l_{2}}\right)$, which is also a ray of the threedimensional Fourier transform $\hat{\phi}$. Each Fourier ray $\boldsymbol{\Lambda}_{k_{1}, l_{1}}$ can be mapped to its direction vector $\boldsymbol{\beta}_{k_{1}, l_{1}}$. The direction vectors of the Fourier rays $\boldsymbol{\Lambda}_{k_{1}, l_{1}}$ and $\boldsymbol{\Lambda}_{k_{2}, l_{2}}$ that correspond to the common line between $P_{k_{1}}$ and $P_{k_{2}}$ must coincide, that is, $\boldsymbol{\beta}_{k_{1}, l_{1}}=\boldsymbol{\beta}_{k_{2}, l_{2}}$. 
polar Fourier transform of the images

$$
\hat{P}_{k}(\rho, \alpha)=\frac{1}{(2 \pi)^{2}} \iint P_{k}(x, y) e^{-i(x \rho \cos \alpha+y \rho \sin \alpha)} d x d y, \quad k=1, \ldots, N,
$$

where $0 \leq \rho<\infty$ and $0 \leq \alpha<2 \pi$. In practice, this is done by fixing an angular resolution $L$, and sampling the Fourier transform (1) along $L$ radial lines, at $n$ equispaced points along each radial line. This results in $L$ vectors $\boldsymbol{\Lambda}_{k, 0}, \ldots, \boldsymbol{\Lambda}_{k, L-1} \in \mathbb{C}^{n}$, given by

$$
\boldsymbol{\Lambda}_{k, l}=\left(\hat{P}_{k}\left(\frac{B}{n}, \frac{2 \pi l}{L}\right), \hat{P}_{k}\left(\frac{2 B}{n}, \frac{2 \pi l}{L}\right), \ldots, \hat{P}_{k}\left(B, \frac{2 \pi l}{L}\right)\right),
$$

where $1 \leq k \leq N, 0 \leq l \leq L-1$ and $B$ is the band limit. Note that the DC term $(\rho=0)$ does not distinguish between lines because it is shared by all lines independently of the image, and is therefore excluded. To determine the common line between two images $P_{i}$ and $P_{j}$, correlations between all $L$ radial lines $\boldsymbol{\Lambda}_{i, l_{1}}$ from the first image with all $L$ radial lines $\boldsymbol{\Lambda}_{j, l_{2}}$ from the second image are computed (overall $L^{2}$ comparisons). The pair of radial lines that has the minimum Euclidean distance (maximum correlation) is declared as the common line. In practice, a weighted Euclidean distance, which is equivalent to applying a combination of high-pass and low-pass filters is used to determine proximity. The "common lines matrix" $C$ is an $N$-by- $N$ array whose $(i, j)$ and $(j, i)$ entries store the indices $l_{1}$ and $l_{2}$, respectively, for which the minimum distance is attained

$$
(C(i, j), C(j, i))=\underset{0 \leq l_{1}<L / 2,0 \leq l_{2}<L}{\operatorname{argmin}}\left\|\boldsymbol{\Lambda}_{i, l_{1}}-\boldsymbol{\Lambda}_{j, l_{2}}\right\| .
$$

In other words, $C(i, j)$ indicates where the $j$ 'th image intersects with the $i$ 'th image. Because different geodesic circles intersect at two antipodal points, it suffices to compute $L^{2} / 2$ correlations.

\section{Voting procedure}

Suppose that the probability of detecting the correct common line between a pair of images is $p$. We assume that detection of common lines is very difficult, but not impossible. In other words, $p \ll 1$, but at the same time $p \gg 2 / L^{2}\left(2 / L^{2}\right.$ can be achieved by choosing the common lines at random). For example, for $p=1 / 5$ only $20 \%$ of the entries in the common lines matrix $C$ in (3) are correct, while the overwhelming majority of $80 \%$ of the entries are false. We now describe a simple voting procedure that discovers the correct common lines.

Consider a pair of projection images $P_{i}$ and $P_{j}$. The common line between projections $i$ and $j$ is insufficient to determine the angle $\alpha_{i j}$ (Figure 2) between their corresponding three-dimensional planes. However, the common lines between three projections $i, j$ and $k$ uniquely determines the relative orientation of the three projections up to handedness (chirality). This is the basis of the angular reconstitution method [van Heel, 1987]. 


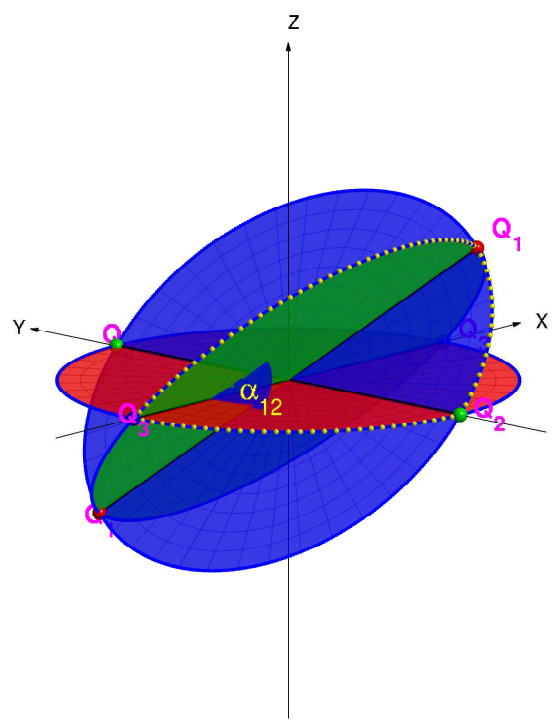

Figure 2: Angular Reconstitution: the common lines between $P_{1}, P_{2}, P_{3}$ uniquely determine the angle $\alpha_{12}$ between $P_{1}$ and $P_{2}$ as well as the three intersection points $Q_{12}$, $Q_{13}$ and $Q_{23}$ ("triangle") of their corresponding great circles on the unit sphere (up to some three-dimensional rotation and possibly a reflection).

First, let us consider the case where the common line between projections $i$ and $j$ was correctly identified. Given the pair $(i, j)$, we consider all $N-2$ different triplets of the form $(i, j, k)(k=1,2, \ldots, N, k \neq i, j)$. Each projection $k$ can vote only once and all votes have equal weight. By the angular reconstitution method, the triplet $(i, j, k)$ determines the angle $\alpha_{i j}$ between projections $i$ and $j$. With probability $p^{2}$ the common lines between projections $i$ and $k$ and between projections $j$ and $k$ are correct. For all such "good" $k$ 's, the resulting angle $\alpha_{i j}$ is the same. With probability $1-p^{2}$ one of the common lines (either $(i, k)$ or $(j, k)$ ) is wrong and the resulting angle $\alpha_{i j}$ is random or non-physical (non-physical common lines are explained in Section 4). There are $p^{2}(N-2)$ "good" third projections on average that all give the same angle. The resulting histogram of the angle $\alpha_{i j}$ is therefore a mixture of a flat distribution (random angles) and a delta-spike at the correct angle. This is demonstrated using simulated data in Section 5, and is illustrated in Figure 5. On the other hand, if the common line between $i$ and $j$ is incorrect, then triplets of the form $(i, j, k)$ give rise to random (or non-physical) angles $\alpha_{i j}$. The histogram of the angle in this case is flat without spikes.

We can distinguish between the two typical histograms (completely random versus random+spike) if the spike is significantly high. In other words, we are able to tell that a common line was correctly identified whenever enough projections voted in the same way. That is, to be able to tell the "good" from the "bad", the spike must consist of 
enough votes, which happens if the condition

$$
p^{2}(N-2) \gg 1
$$

is satisfied. This shows that even at low detection rates, the larger the data set the better. For example, when $p=1 / 5$ and $N=10000$ we expect a spike of size $\approx 400$.

In practice, we have no estimate for the value of $p$. Instead, we plot for each pair of projections its angle histogram, and record the height of its peak. Following the discussion above, even though we do not know $p$, the angle histogram for pairs for which the common line was correctly identified will exhibit a higher peak than for pairs for which the common line was misidentified. This is true as long as $p$ is not too small. Thus, once we compute the peak of the angle histogram for each pair of projections, we plot the histogram of the peaks. Pairs of projections that correspond to the righthand-side of the peaks histogram are those for which the peak of their angle histogram was highest. It is thus more likely that the common line between those projections was correctly identified. We demonstrate this in Section 5. For explanatory purposes, we assume in the simulations in Section 5 that $p$ is known, to demonstrate quantitatively the performance of the algorithm. This is not required when processing experimental data.

\section{Disqualified votes}

Not all triplets of common lines can be realized as planes whose common lines are the given triplet. Such inconsistent triplets lead to non-physical angles, as we now explain. As illustrated in Figure 2, the three great circles corresponding to projections 1, 2 and 3 intersect on the unit sphere at $Q_{12}, Q_{13}$ and $Q_{23}\left(Q_{i j}\right.$ is the intersection of the two circles corresponding to projections $i$ and $j$; there are also three antipodal intersection points). The three common lines determine the distances between the three intersection points. Those distances are always between 0 and 2 (the largest distance between points on the unit sphere). For three distances to form a triangle, they must satisfy the triangle inequality. It turns out that the triangle inequality is not sufficient to determine the triangle, because the three points must lie on the unit sphere as well. For example, the distances 2,2,2 satisfy the triangle inequality, but the corresponding triangle is too big to be placed on the unit sphere. The exact condition that guarantees a successful triangulation is obtained by using either linear algebra or geometry. We first give the linear algebra derivation.

The three dot products between the three points $Q_{i j}, Q_{i k}$, and $Q_{j k}$ are obtained from the common lines between projections $i, j$, and $k$ by

$$
\left\langle Q_{i j}, Q_{i k}\right\rangle=\cos (2 \pi(C(i, j)-C(i, k)) / L)
$$

where $C(i, j)$ is the index of the common line between projections $i$ and $j$ at the plane

of projection $i$. Since the points are on the unit sphere, we have $\left\langle Q_{i j}, Q_{i j}\right\rangle=1$. The 
Gram matrix of $Q_{12}, Q_{13}, Q_{23}$ is the 3-by-3 matrix of their dot products given by

$$
\left(\begin{array}{ccc}
1 & \left\langle Q_{23}, Q_{13}\right\rangle & \left\langle Q_{23}, Q_{12}\right\rangle \\
\left\langle Q_{13}, Q_{23}\right\rangle & 1 & \left\langle Q_{13}, Q_{12}\right\rangle \\
\left\langle Q_{12}, Q_{23}\right\rangle & \left\langle Q_{12}, Q_{13}\right\rangle & 1
\end{array}\right)=\left(\begin{array}{ccc}
1 & a & b \\
a & 1 & c \\
b & c & 1
\end{array}\right)
$$

where

$$
\begin{aligned}
& a=\cos (2 \pi(C(3,2)-C(3,1)) / L), \\
& b=\cos (2 \pi(C(2,3)-C(2,1)) / L), \\
& c=\cos (2 \pi(C(1,3)-C(1,2)) / L) .
\end{aligned}
$$

We define

$$
G=\left(\begin{array}{lll}
1 & a & b \\
a & 1 & c \\
b & c & 1
\end{array}\right)
$$

Note that the matrix $G$ in (8) can always be formed by combining the common lines information with (7). We want to find a condition under which there exist coordinates $Q_{12}, Q_{13}, Q_{23}$ such that (6) holds. A necessary and sufficient condition in that the matrix (8) is positive definite. To see this, suppose that we can write $G$ in (8) as a matrix of dot products as in (6). Then, $G=Q^{T} Q$ where $Q=\left(Q_{23}, Q_{13}, Q_{12}\right)$ is the matrix having the coordinates of $Q_{23}, Q_{13}$, and $Q_{12}$ as its columns, which immediately shows that $G$ is positive definite. Conversely, if $G$ is positive definite, then the Cholesky decomposition [Golub and Van Loan, 1984] of $G$ is in the form of $G=Q^{T} Q$ and so (6) holds. We proceed to derive the condition for $G$ to be positive definite.

We begin with examining the trace of $G$

$$
\operatorname{Tr}(G)=3=\lambda_{1}+\lambda_{2}+\lambda_{3}
$$

where $\lambda_{1} \geq \lambda_{2} \geq \lambda_{3}$ are the sorted eigenvalues of $G$, which immediately implies $\lambda_{1}>0$. Since $|a|,|b|,|c| \leq 1$ it follows that the sums of the absolute values of the rows of $G$ are bounded by $3: 1+|a|+|b| \leq 3,1+|a|+|c| \leq 3$ and $1+|b|+|c| \leq 3$. By the Gershgorin circle theorem [Golub and Van Loan, 1984] it follows that $\lambda_{1} \leq 3$. Combining this with (9) we obtain that $\lambda_{2}+\lambda_{3} \geq 0$. Therefore, $\lambda_{2} \geq 0$ (because $2 \lambda_{2} \geq \lambda_{2}+\lambda_{3} \geq 0$ ). A necessary and sufficient condition for positive definiteness is that all eigenvalues are positive. Since we have already established that $\lambda_{1} \geq \lambda_{2} \geq 0$, it remains to require that the smallest eigenvalue is positive, that is, to require that $\lambda_{3}>0$. To that end, we use the determinant of $G$ which equals the product of the eigenvalues: $\operatorname{det}(G)=\lambda_{1} \lambda_{2} \lambda_{3}$. In our case, the determinant is given by

$$
\operatorname{det}(G)=1-\left(a^{2}+b^{2}+c^{2}\right)+2 a b c
$$

We conclude that the condition for positive definiteness is

$$
1+2 a b c>a^{2}+b^{2}+c^{2}
$$


The condition (11) explains for example why the distances 2,2,2 corresponding to $a=b=c=-1$ are not realizable on the sphere. Only triplets $(i, j, k)$ that satisfy the condition (11) are physical and eligible to vote. All other votes are disqualified. Though it may be tempting to think that condition (11) is violated only when projections

are nearby and their common lines lie very close to each other and therefore are not informative anyway, even moderate angles, such as $a=b=c=-1 / 2$ lead to violations.

An alternative approach for deriving condition (11) uses the geometry of the sphere. We may assume that the circle corresponding to projection 1 lies in the $x y$-plane, so it has the parametrization $\left(\cos \theta_{1}, \sin \theta_{1}, 0\right)\left(0 \leq \theta_{1}<2 \pi\right)$. By an arbitrary choice of the coordinate system, the intersection point of projections 1 and 2 is

$$
Q_{12}=(1,0,0)
$$

and the intersection point of projections 1 and 3 is

$$
Q_{13}=\left(c, \sqrt{1-c^{2}}, 0\right) \text {. }
$$

Since the great circle that corresponds to projection 2 goes through $Q_{12}=(1,0,0)$, it follows that its parametrization is given by $\left(\cos \theta_{2}, \cos \alpha_{12} \sin \theta_{2}, \sin \alpha_{12} \sin \theta_{2}\right)\left(0 \leq \theta_{2}<\right.$ $2 \pi$ ), where $\alpha_{12}$ is the angle between projections 1 and 2 (see Figure 2). In particular, from $\left\langle Q_{12}, Q_{23}\right\rangle=b$, we get

$$
Q_{23}=\left(b, \sqrt{1-b^{2}} \cos \alpha_{12}, \sqrt{1-b^{2}} \sin \alpha_{12}\right) .
$$

Taking the dot product between $Q_{13}$ and $Q_{23}$ we obtain

$$
\begin{aligned}
a & =\left\langle Q_{13}, Q_{23}\right\rangle=\left(c, \sqrt{1-c^{2}}, 0\right) \cdot\left(b, \sqrt{1-b^{2}} \cos \alpha_{12}, \sqrt{1-b^{2}} \sin \alpha_{12}\right) \\
& =b c+\sqrt{1-b^{2}} \sqrt{1-c^{2}} \cos \alpha_{12}
\end{aligned}
$$

from which $\cos \alpha_{12}$ is extracted

$$
\cos \alpha_{12}=\frac{a-b c}{\sqrt{1-b^{2}} \sqrt{1-c^{2}}}
$$

The condition $\cos ^{2} \alpha_{12} \leq 1$ is equivalent to (11). The voting algorithm is outlined in Algorithm 1.

\section{Results}

We conducted several numerical experiments to test the performance of the voting procedure. In Section 5.1 we apply the algorithm on simulated electron-microscope projections. This allows us to demonstrate quantitatively the performance of the algorithm. Then, in Section 5.2, we apply the algorithm on a real electron microscope data set, obtaining three-dimensional models directly from a large number of class averages. 


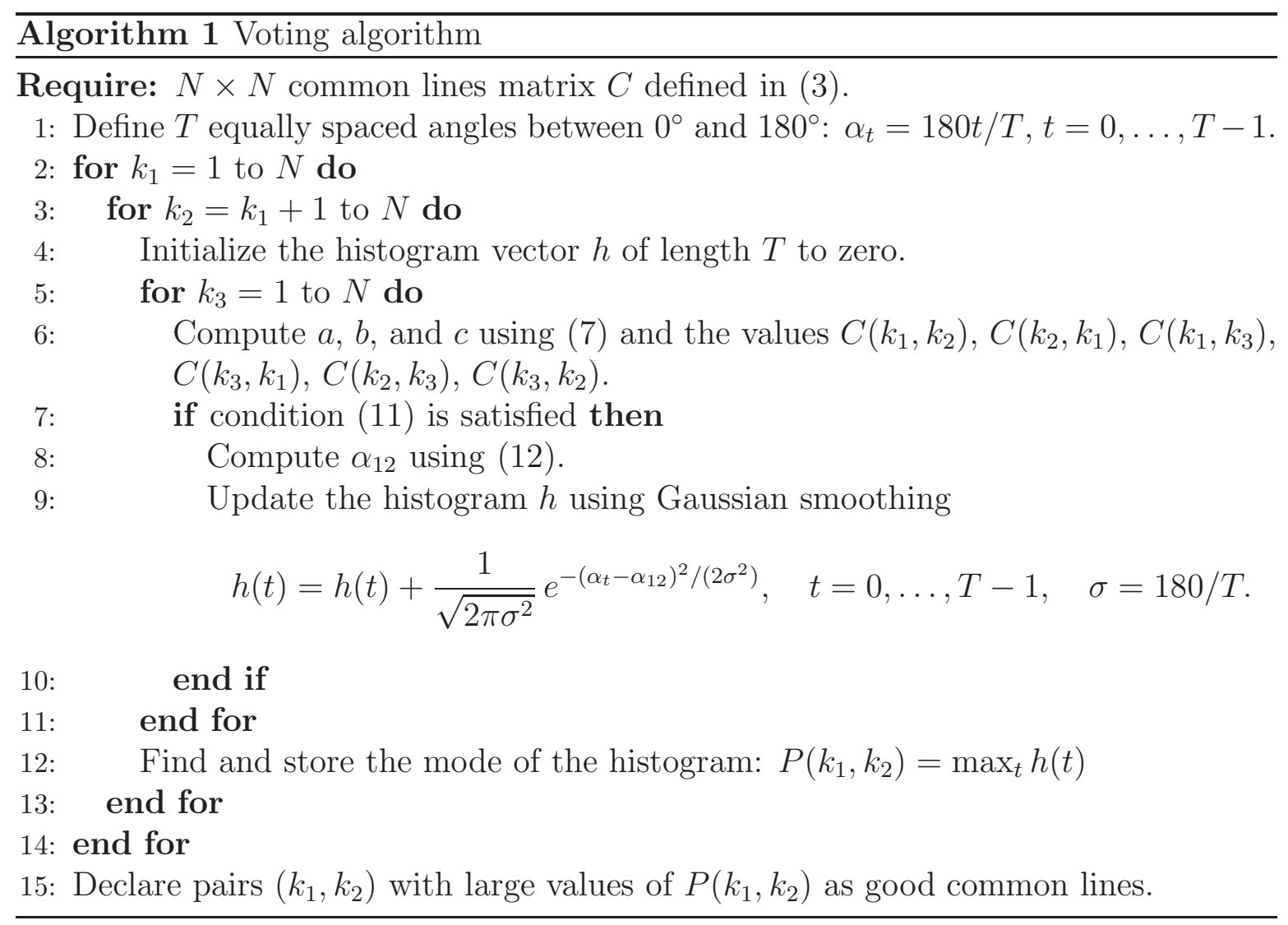




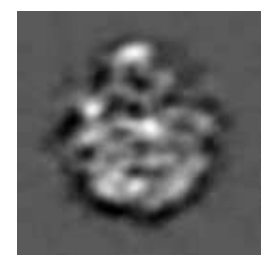

(a) Clean

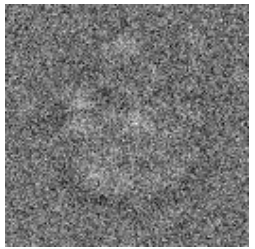

(f) $\mathrm{SNR}=1 / 16$

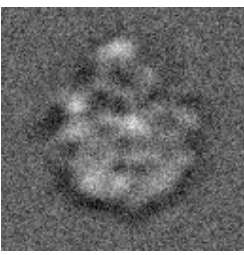

(b) $\mathrm{SNR}=1$

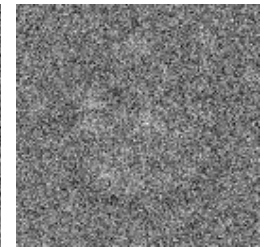

(g) $\mathrm{SNR}=1 / 32$

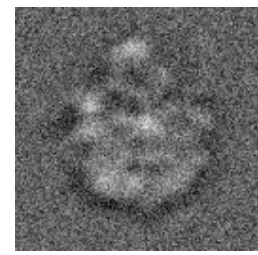

(c) $\mathrm{SNR}=1 / 2$

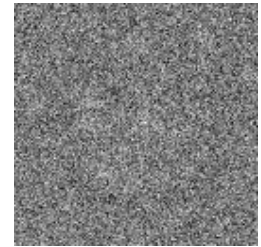

(h) $\mathrm{SNR}=1 / 64$

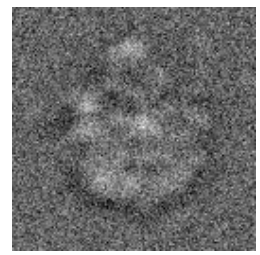

(d) $\mathrm{SNR}=1 / 4$

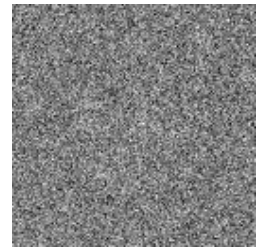

(i) $\mathrm{SNR}=1 / 128$

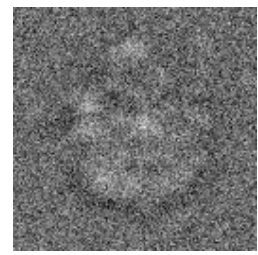

(e) $\mathrm{SNR}=1 / 8$

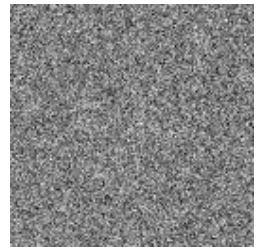

(j) $\mathrm{SNR}=1 / 256$

Figure 3: Simulated projection with various levels of additive Gaussian white noise.

\subsection{Simulations}

We applied the voting algorithm on sets of simulated projections of a ribosome, containing $N=100,500,1000$, and 5000 projections. For each $N$, we generated $N$ noisefree centered projections of the ribosome at uniformly distributed random orientations. Specifically, the projection orientations were obtained by sampling the set of all threedimensional rotations, known as the rotation group $\mathrm{SO}(3)$, uniformly at random. Each projection was of size $129 \times 129$ pixels. Next, we fixed a signal-to-noise ratio (SNR), and added to each clean projection additive Gaussian white noise of the prescribed SNR. The SNR in all our experiments is defined by

$$
\mathrm{SNR}=\frac{\operatorname{Var}(\text { Signal })}{\operatorname{Var}(\text { Noise })},
$$

where Var is the variance (energy), Signal is the clean projection image and Noise is the noise realization of that image. Figure 3 shows one of the projections at different SNR levels. The SNR values used throughout this experiment were $2^{-k}$ with $k=0, \ldots, 9$. Clean projections were generated by setting $\mathrm{SNR}=2^{20}$.

The first step of the experiment was to determine the angular resolution $L$. The algorithm is faster with small values of $L$ since the number of correlations that need to be computed in order to detect a common line is $L^{2} / 2$. On the other hand, too small of an $L$ will prevent common line routines from detecting a good approximation of the true common line due to poor angular resolution. In all subsequent experiments we use $L=72$, which corresponds to an angular resolution of $5^{\circ}$.

Once $L$ has been fixed, we took sets of noisy projections with a given SNR, and constructed for each set its corresponding common lines matrix. The percentage of correctly identified common lines in each matrix is plotted against the SNR for various 
values of $N$ in Figures $4 \mathrm{a}-4 \mathrm{~d}$, using the curve designated by "no filtering". Each such curve gives the probability $p$ of detecting common lines between projections as a function of the SNR. In all experiments we consider the common lines between two projections as correctly identified, if the estimated common lines deviate from the true ones by up to $10^{\circ}$.

We then applied "correlation filtering" to the common lines matrices, that is, we retained only common lines whose correlations are among the highest $p$ percentile of correlations. Specifically, we retained a common line $\left(\boldsymbol{\Lambda}_{i, l_{1}}, \boldsymbol{\Lambda}_{j, l_{2}}\right)$ only if the correlation between rays $\boldsymbol{\Lambda}_{i, l_{1}}$ and $\boldsymbol{\Lambda}_{j, l_{2}}$ is one of the highest $p\left(\begin{array}{c}N \\ 2\end{array}\right)$ correlations. We then plotted the percentage of correct common lines among the retained common lines. This is shown in Figures 4a-4d using the curve designated by "correlation filtering". Obviously, this filtering improves the detection rate of common lines. Note that since there are only $p\left(\begin{array}{c}N \\ 2\end{array}\right)$ correct common lines, there is no point in retaining more than that, as any larger number would necessarily increase the number of errors.

Finally, we filtered the original common lines matrices using the voting procedure, which we also refer to as "histogram filtering". The histogram filtering consists of several steps. The first step is to compute for each pair of projections $\left(P_{i}, P_{j}\right)$ the angle induced between them by all third projections. This gives a series of $N-2$ estimates for the angle $\alpha_{i j}$ between $P_{i}$ and $P_{j}$. If the common line between projections $P_{i}$ and $P_{j}$ was correctly identified, we expect these estimates to be centered around the true angle between $P_{i}$ and $P_{j}$. That is, we expect the histogram of the estimates to exhibit a peak at the correct angle.

To find this peak, we use a Gaussian kernel to obtain a smooth density estimation for the angle between $P_{i}$ and $P_{j}$, followed by mode seeking over a discrete set of $T=60$ equally spaced angles between $0^{\circ}$ to $180^{\circ}$. We choose the width of the Gaussian kernel as $\sigma=3^{\circ}$ (see also steps 1 and 9 in Algorithm 1). The Gaussian smoothing serves as a simple way for binning the histogram such that close-by angle estimates are combined into a single peak. In Figure 5 we show several examples for the smoothed histogram of the angle between pairs of projections. These histograms were obtained from the experiment that corresponds to $N=1000$ and $\mathrm{SNR}=1 / 16$. Figures 5a-5d show smoothed histograms for pairs of projections where the common lines were correctly identified. Figures $5 \mathrm{e}-5 \mathrm{~h}$ correspond to pairs of projections for which the common lines were misidentified. Note the different scaling of the $y$-axis between the cases of correct and incorrect identification of common lines.

As explained at the end of Section 3, we record for each angle histogram the height of its peak (step 12 in Algorithm 1), and compute the histogram of the peaks. We then retain only common lines whose peaks are among the $p$ percentile of highest peaks ( $p$ is the probability of detecting a correct common line, obtained from the curve designated by "no filtering" in Figures $4 \mathrm{a}-4 \mathrm{~d}$ ). The resulting percentage of correct common lines is shown in Figures 4a-4d using the curve designated by "histogram filtering". As evident from Figures $6 \mathrm{a}-6 \mathrm{c}$, increasing $N$ improves the performance the histogram filtering, but not of the correlation filtering. However, as Figures $4 \mathrm{a}-4 \mathrm{~d}$ show, histogram filtering is consistently superior to correlation filtering. 


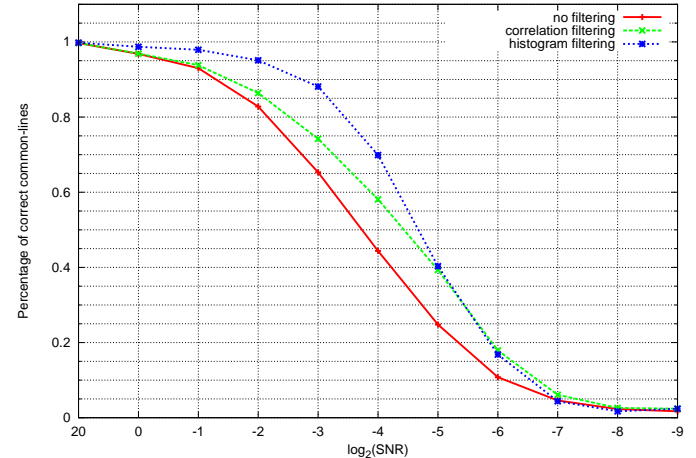

(a) $N=100$

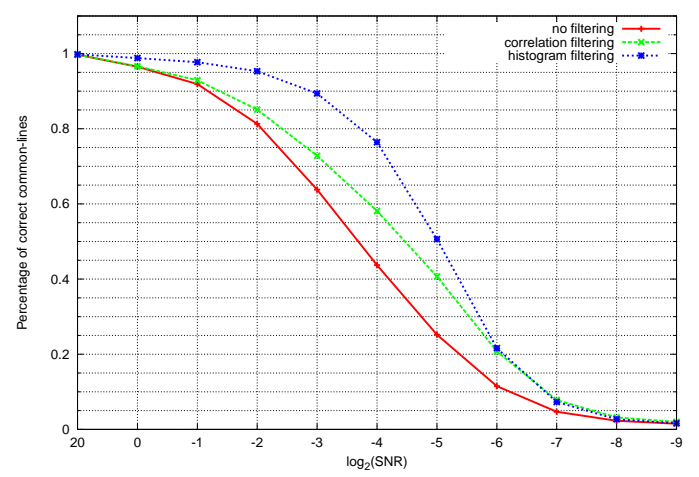

(c) $N=1000$

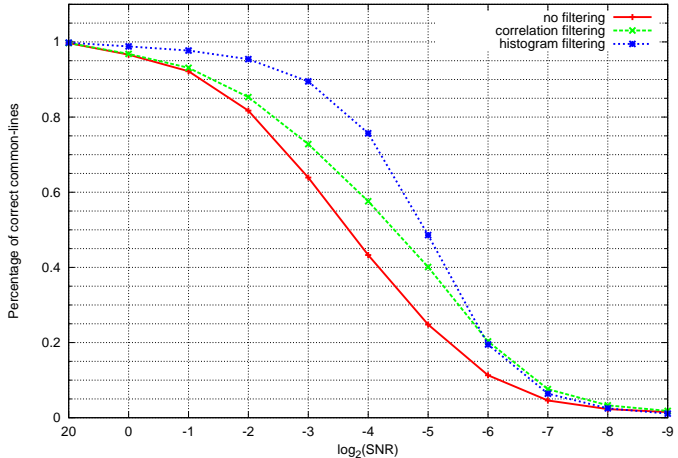

(b) $N=500$

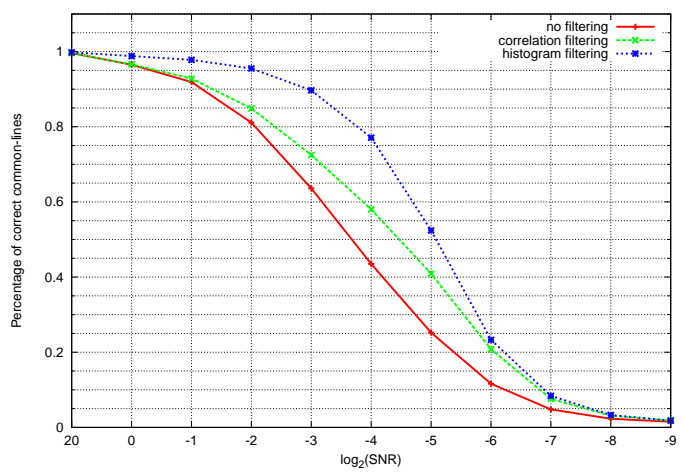

(d) $N=5000$

Figure 4: Comparing correlation filtering and histogram filtering for (a) $N=100$, (b) $N=500$, (c) $N=1000$, (d) $N=5000$. 


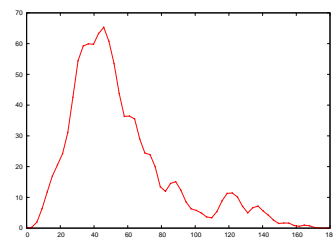

(a) $i=18, j=481$

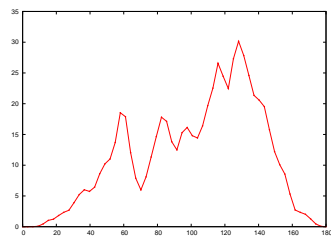

(e) $i=18, j=491$

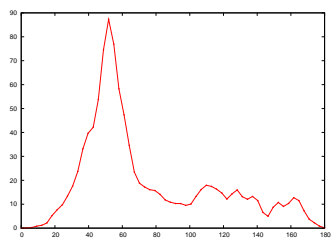

(b) $i=177, j=616$

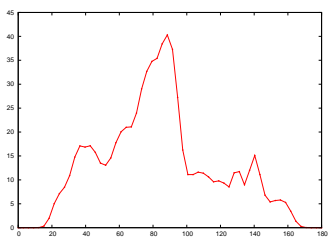

(f) $i=105, j=520$

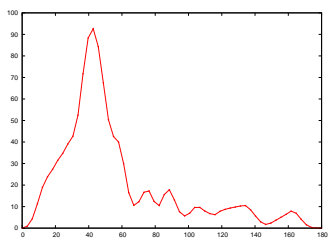

(c) $i=394, j=955$

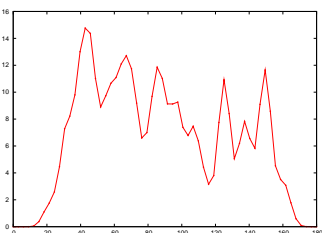

(g) $i=822, j=860$

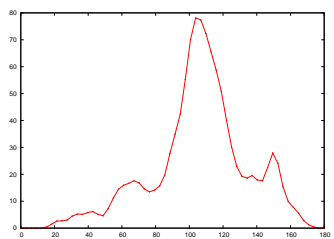

(d) $i=802, j=884$

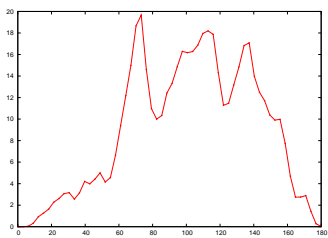

(h) $i=899, j=978$

Figure 5: Smoothed histograms of the angle (in degrees) between pairs of projections. The plots were generated using $N=1000$ projections with $\mathrm{SNR}=1 / 16$. Top row corresponds to pairs of projections whose common lines were correctly identified. Bottom row corresponds to pairs of projections whose common lines were misidentified. Note the different scale of the $y$-axis in the two cases, indicating much higher peaks for correctly identified common-lines.

Figures $7 \mathrm{a}-7 \mathrm{j}$ show the histograms of peaks for $N=1000$ projections and various levels of SNR. As can be seen from the figures, for lower noise levels (see for example Figures $7 \mathrm{~d}$ and $7 \mathrm{e}$ ), the histograms consist of two well-separated distributions (bumps) - the right peak corresponds to the average peak height of histograms of correctly identified common lines; the left peak corresponds to the average peak height of histograms of misidentified common lines. As the noise level increases, the two distributions start to overlap. Figures $8 \mathrm{a}-8 \mathrm{~d}$ show the histogram of peaks for a fixed $\mathrm{SNR}=1 / 16$ and various values of $N$. As $N$ increases, it becomes possible to resolve the peak that corresponds to misidentified common lines from the peak that corresponds to correctly identified common lines.

\subsection{Reconstruction from ribosome images}

A set of micrographs of E. coli 50S ribosomes was provided by M. van Heel. These images were acquired with a Philips CM20 at defocus values between 1.37 and 2.06 $\mu \mathrm{m}$; they were scanned at $3.36 \AA$ /pixel, and particles were picked using the automated particle picking algorithm in EMAN Boxer. All subsequent image processing was performed with the IMAGIC software package [Stark, H. et al., 2002; van Heel, M. et al., 1996]. The particle images were phase-flipped to remove the phase-reversals in the CTF and bandpass filtered at $1 / 150 \AA$ and $1 / 8.4 \AA$. The variance-normalized images were translationally aligned with the rotationally-averaged total sum.

Without rotational alignment, the 27,121 particle images were classified using the 


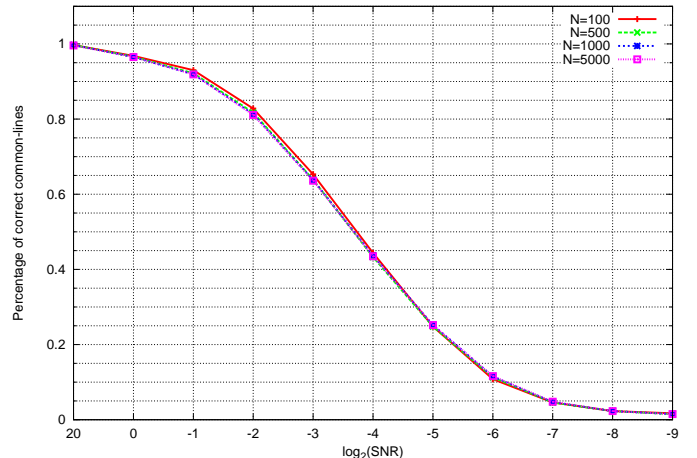

(a) No filtering

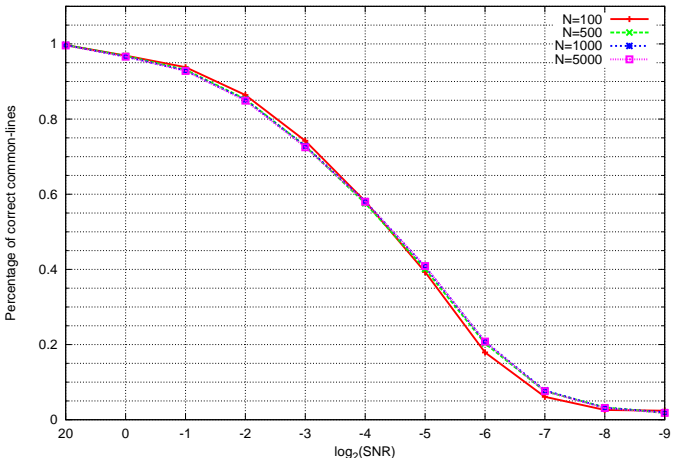

(b) Correlation filtering

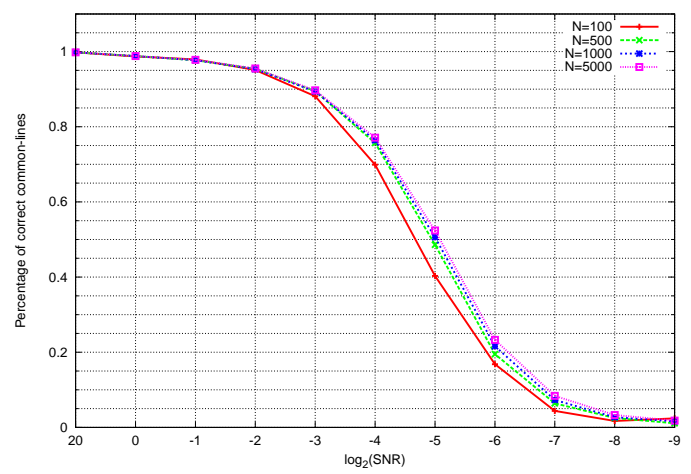

(c) Histogram filtering

Figure 6: Common lines detection rate as a function of the SNR, for various values of $N$, when using (a) no filtering, (b) correlation filtering, (c) histogram filtering. 


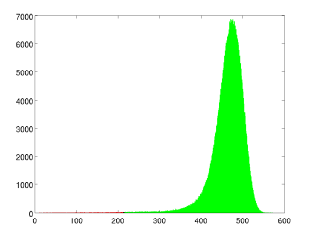

(a) Clean projections

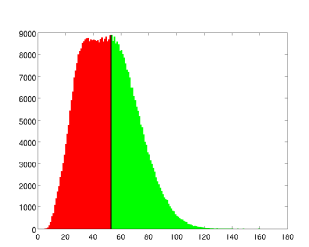

(f) $\mathrm{SNR}=1 / 16$

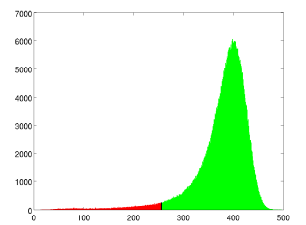

(b) $\mathrm{SNR}=1$

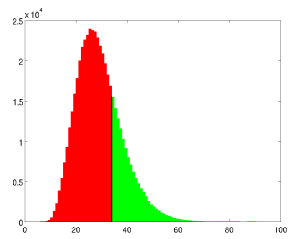

(g) $\mathrm{SNR}=1 / 32$

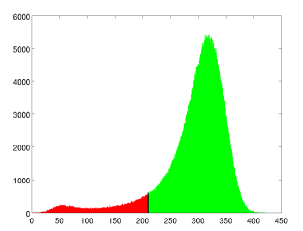

(c) $\mathrm{SNR}=1 / 2$

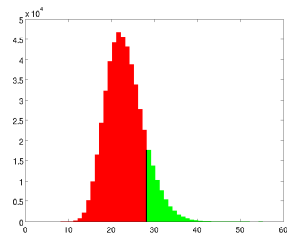

(h) $\mathrm{SNR}=1 / 64$

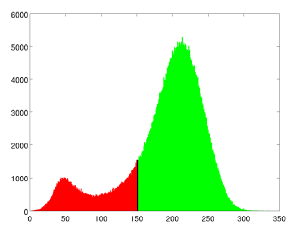

(d) $\mathrm{SNR}=1 / 4$

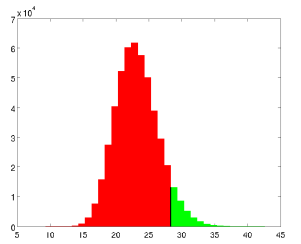

(i) $\mathrm{SNR}=1 / 128$

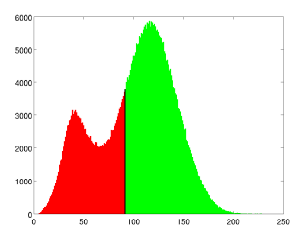

(e) $\mathrm{SNR}=1 / 8$

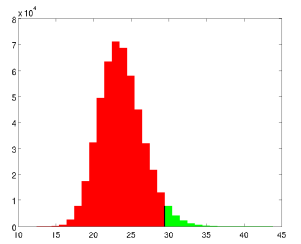

(j) $\mathrm{SNR}=1 / 256$

Figure 7: Histogram of peaks for $N=1000$ and various levels of noise. The top $p$ percentile of each histogram is marked in Green, the bottom $1-p$ percentile is marked in Red, and the boundary between the regions is marked as a black vertical line. The area of the Green region corresponds to the number of correct entries in the commonlines matrix. The location of the boundary is the minimal peak height to be considered by the algorithm as a correctly identified common line. Note how this threshold value decreases as the SNR decreases. The algorithm assumes that the correct common lines are concentrated in the Green area and that the wrong common lines are concentrated in the Red area.

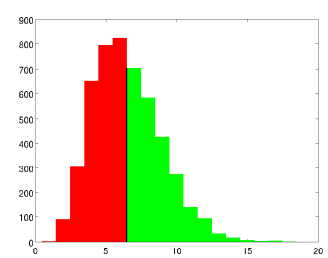

(a) $N=100$

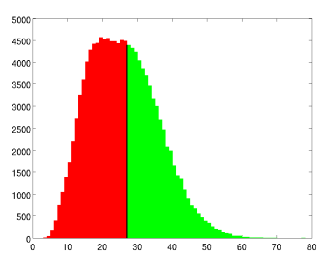

(b) $N=500$

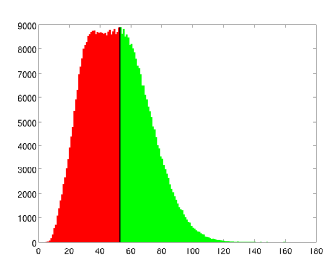

(c) $N=1000$

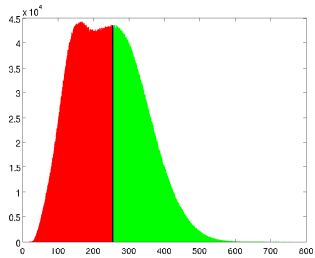

(d) $N=5000$

Figure 8: Histogram of peaks for $\mathrm{SNR}=1 / 16$ for $N=100,500,1000,5000$. 
MSA function into sets of 750, 1500 and 3000 classes, and the class means were used for the voting algorithm. In parallel, the IMAGIC routines were used to perform multiple cycles of multireference alignment and classification, reconstruction using angular reconstitution, and model refinement. The refined model had a resolution of about $11.7 \AA$.

A comparison of the refined model and the three models obtained directly from the sets of 3000, 1500 and 750 class averages is shown in Figure 9. The 750 class averages yielded the lowest-quality reconstruction because the number of classes does not sufficiently sample the three Euler angles of orientation. Figure 10 shows the corresponding Fourier shell correlations (FSCs). The FSC of the refined model, obtained from reconstructions of two halves of the data set, shows a nominal resolution of about $15 \AA$ at the 0.5 threshold criterion and $11.7 \AA$ using the 3 -sigma criterion. FSCs were also computed between the refined model and the models obtained directly from the voting algorithm. In that single step, models with resolutions of about $25 \AA$ were obtained, with the 1500 and 3000-class reconstructions being slightly better than the 750-class reconstruction. The algorithm failed when using 500 class averages due to excessive averaging, and for 5000 and 7000 class averages due to the low detection rate of common lines.

In examining the structures in Figure 9, it is evident that the structures obtained from the common lines approach (Figures 9b-9d) illustrate a remarkably strong correspondence to that generated via the Imagic 3D reconstruction approach (Figure 9a). There are, however, structural features that are not well resolved by the common lines approach (black arrows). As stated earlier, the benefit of the common lines approach is that multiple rounds of reference based alignment of the particle database are not required to obtain the starting ab initio structure. Moreover, the use of a reference based bias in arriving at a starting structure for refinement is not an issue in the common lines approach since the starting assumption is that all projections of a given $3 \mathrm{D}$ volume are self-consistent with that structure. As a result, the common lines approach benefits from the fact that as the numbers of class averages are increased such that fewer particles participate per class, there is an inherent improvement in the variance/member. Conversely, in the smaller class average data sets, the potential increased variance in angular distribution within a class would result in the loss of our ability to resolve important structural features, as is the case with the traditional approaches. Without proper refinement protocols in the traditional 3D reconstruction approach, it is possible that significant errors in Euler angle assignment could lead to a 'wrong' structure. The common lines approach ameliorates this as a potential issue.

As the input class averages increase in number, the number of participating particles per class decreases from $\approx 30$ to 7 as we move from 750 to 3000 classes, respectively. Several interesting features arise from a comparison of these structures generated by the common lines approach. It is clearly obvious that, as a result of a larger range of angular displacement, the structures derived from the small number of input classes results in less structural definition as would be expected. However, this is true even of the 3000 input class structure. Some of the finer elements (black arrows) are not 


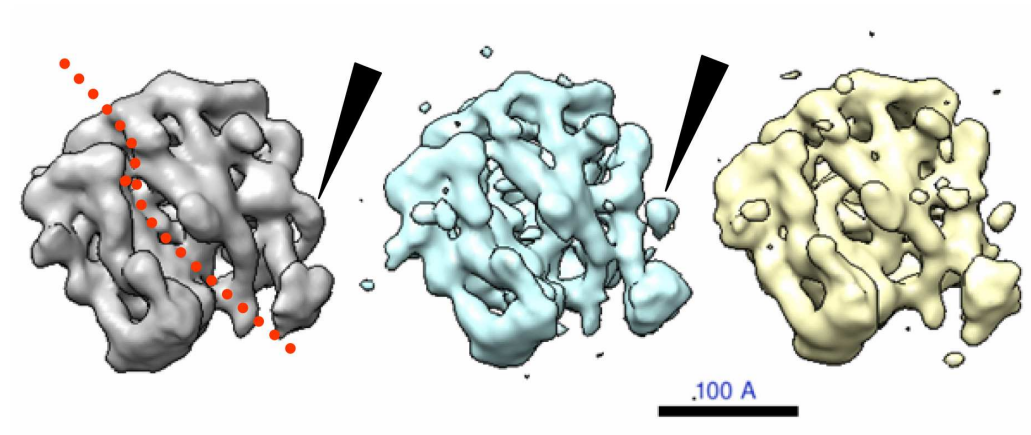

(a) Refined

(b) $N=3000$

(c) $N=1500$

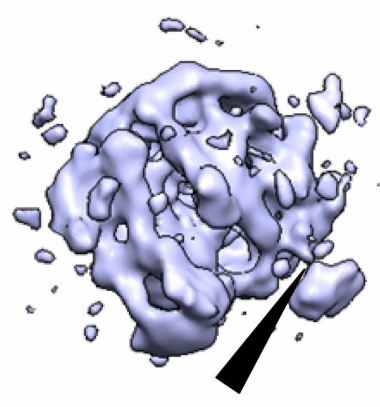

(d) $N=750$

Figure 9: Differences in the capacity of the common lines approach to readily define a starting structure for continued refinement. Figure (a) was generated from an Imagic reference-based alignment of the particle data set used in this study and refined to $11.7 \AA$ resolution. The RNA binding groove is shown by the red dotted chain. This structure is comparable to that currently published, and was generated to evaluate the relative Fourier Shell Correlation of structures derived from the voting approach as a function of numbers of input class averages. The remaining figure was generated from the voting algorithm using (b) 3000, (c) 1500, and (d) 750 input classes. The voting-based structures, for the sake of comparison, were soft masked and filtered to $15 \AA$ resolution. The structures were also flipped about the $z$-axis such that their handedness is consistent with that published [Avila-Sakar, A. J. et al., 1994] and shown in the Imagic generated 3D volume.

resolved. There are also some internal chains which are incomplete. One major factor that is of significant interest, however, is that the RNA binding groove appears to be significantly deeper in these structures. This is interesting in light of the locations of the A, P and E catalytic and release sites as well as RNA binding within this 50S ribosome. While it is outside the scope of this manuscript, it would be interesting to dock some of the crystal structures determined for these domains and see if a deeper binding groove better accommodates the various elements involved in the protein synthetic process. When looking at the crystal structure of [Avila-Sakar, A. J. et al., 1994] and [Mitra, K. et al., 2006] shown in Figure 11, it would appear that a deeper RNA binding groove may prove more reasonable.

\section{Summary and discussion}

We presented a simple and efficient voting procedure that makes use of the geometry rendered by the Fourier projection-slice theorem to identify the correct common lines even in the presence of many other falsely detected common lines. The quality of a common line is determined by all other images. Our method succeeds even at a low detection rate of common lines and would therefore allow common lines-based methods 


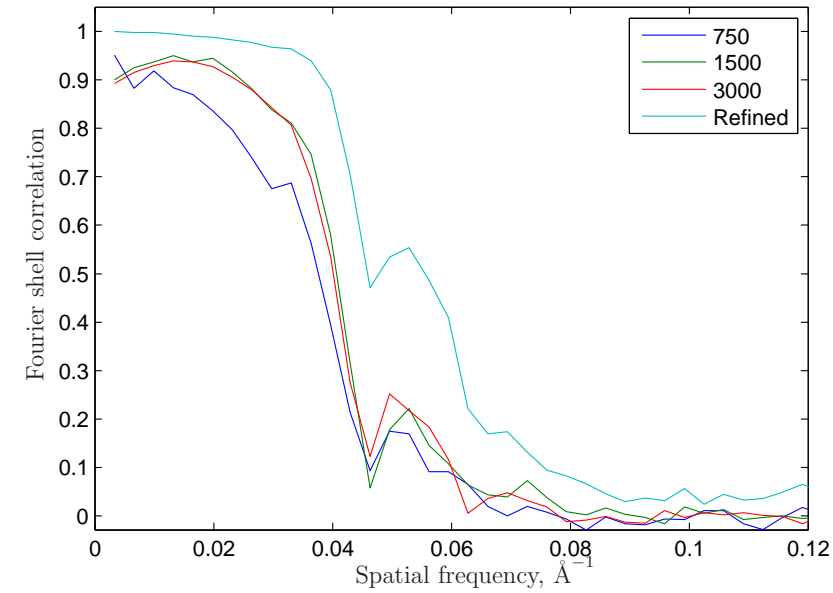

Figure 10: Fourier shell correlations of the various reconstructions.

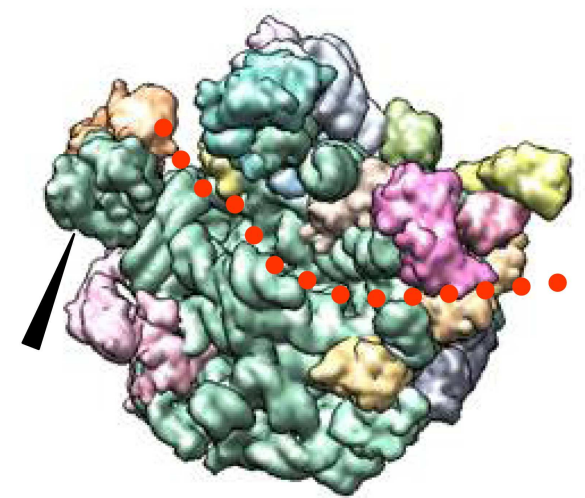

Figure 11: A $3 \AA$ surface rendering of the ribosomal 50 S subunit generated by [Mitra, K. et al., 2006]. The upper stalk (arrow) is the same stalk region in the cryo-EM structures shown in Figure 9 and functions in protein chain elongation and ejection of the nascent t-RNA from the E site. The RNA binding groove is also shown (red dotted lace). 
to succeed in lower SNR. It would allow, for example, to use noisier class averages, where each class consists of fewer projections.

The voting procedure can be easily adjusted to handle cases in which there are several common line candidates: for each candidate we produce a histogram and choose the one (if any) that shows an identifiable spike.

We note that the method may also be useful for the heterogeneity problem. In theory, if we pick a pair of projections corresponding to different types, then all triplets containing the pair should be incoherent and produce random angles. In practice, however, the problem of heterogeneity is more difficult. Projections of different types are very similar and can easily fool the common line test. This is especially true when dealing with class averages that may contain projections from different types.

Our experience with simulative data shows that the detection rate of common lines between a fixed pair of images exhibits a phase transition behavior. Once the SNR goes below a certain threshold, the detection rate decays exponentially quickly. This is in agreement with the threshold phenomenon in non-linear estimation theory that was developed originally for radar range estimation [Zakai, M. and Ziv, J., 1969; Ziv, J. and Zakai, M., 1969]. In our case, the threshold is different from one pair of images to the other, and so some common lines may be correctly detected while others are not. In other words, although the detection of common lines between a fixed pair of images exhibits a sharp threshold phenomenon as a function of the SNR, the threshold region is much wider and smoother for the entire data set since we are comparing many different pairs with different thresholds.

Improved filters and correlation tests for common line detection can push the detection threshold lower and therefore significantly improve the performance of any common lines based algorithm like the one presented in this paper. We believe that constructing improved comparison tests, such as developing tests based on some clever feature selection to replace simple correlation, is a research direction that should be strongly pursued.

Finally, we note that although the voting procedure finds the correct common lines, it may happen that it wrongly detects false common lines as being correct. Such outliers may be post-identified using the energy minimization procedure of [Penczek et al., 1996]. We are currently developing an alternative spectral method that shows potential of handling a larger percentage of outliers. This spectral method will be reported in a separate publication.

\section{References}

Avila-Sakar, A. J., Guan, T. -L., Arad, T., Schmid, M. F., Loke, T. W., Yonath, A., Piefke, J., Franceschi, F., Chiu, W. J., 1994. Electron cryomicroscopy of bacillus stearothermophilus $50 \mathrm{~S}$ ribosomal subunits crystallized on phospholipid monolayers. Molecular Biology 239, 689-697.

Chiu, W., Baker, L., M., Jiang, W., Dougherty, M., Schmid, M. F., 2005. Electron 
cryomicroscopy of biological machines at subnanometer resolution. Structure 13 (3), 363-372, review. PMID: 15766537 [PubMed - indexed for MEDLINE].

Farrow, M., Ottensmeyer, P., 1992. A posteriori determination of relative projection directions of arbitrarily oriented macrmolecules. Journal of the Optical Society of America A: Optics, Image Science, and Vision 9 (10), 1749-1760.

Frank, J., 2006. Three-Dimensional Electron Microscopy of Macromolecular Assemblies: Visualization of Biological Molecules in Their Native State. Oxford.

Golub, G. H., Van Loan, C. F., 1984. Matrix Computation. Johns Hopkins series in the mathematical sciences. The Johns Hopkins University Press.

Henderson, R., 2004. Realizing the potential of electron cryo-microscopy. Q Rev Biophys 37 (1), 3-13, review. PMID: 17390603 [PubMed - indexed for MEDLINE].

Ludtke, S. J., Baker, M. L., Chen, D. H., Song, J. L., Chuang, D. T., Chiu, W., 2008. De novo backbone trace of GroEL from single particle electron cryomicroscopy. Structure 16 (3), 441-448.

Mallick, S. P., Agarwal, S., Kriegman, D. J., Belongie, S. J., Carragher, B., Potter, C. S., 2006. Structure and view estimation for tomographic reconstruction: A Bayesian approach. Computer Vision and Pattern Recongnition (CVPR) II, 2253-2260.

Mitra, K., Schaffitzel, C., Fabiola, F., Chapman, M. S., Ban, N., Frank, J., 2006. Elongation arrest by SecM via a cascade of ribosomal RNA rearrangements. Molecular Cell 22, 533-543.

Natterer, F., 2001. The Mathematics of Computerized Tomography. Classics in Applied Mathematics. SIAM: Society for Industrial and Applied Mathematics.

Penczek, P. A., Zhu, J., Frank, J., 1996. A common-lines based method for determining orientations for $N>3$ particle projections simultaneously. Ultramicroscopy 63, 205218.

Stark, H., Rodnina, M. V., Wieden, H. J., Zemlin, F., Wintermeyer, W., van Heel, M., 2002. Ribosome interactions of aminoacyl-tRNA and elongation factor $\mathrm{Tu}$ in the codon-recognition complex. Nature Structural Biology 9 (849-854).

Vainshtein, B., Goncharov, A., 1986. Determination of the spatial orientation of arbitrarily arranged identical particles of an unknown structure from their projections. In: Proc. llth Intern. Congr. on Elec. Mirco. pp. 459-460.

van Heel, M., 1987. Angular reconstitution: a posteriori assignment of projection directions for 3D reconstruction. Ultramicroscopy 21 (2), 111-123, pMID: 12425301 [PubMed - indexed for MEDLINE]. 
van Heel, M., Harauz, G., Orlova, E. V., Schmidt, R., Schatz, M., 1996. A new generation of the IMAGIC image processing system. Journal of Structural Biology 116 (1), $17-24$.

Wang, L., Sigworth, F. J., 2006. Cryo-em and single particles. Physiology (Bethesda) 21, 13-18, review. PMID: 16443818 [PubMed - indexed for MEDLINE].

Zakai, M., Ziv, J., 1969. On the threshold effect in radar range estimation. IEEE Transactions on Information Theory 15 (1), 167-170.

Zhang, X., Settembre, E., Xu, C., Dormitzer, P. R., Bellamy, R., Harrison, S. C., Grigorieff, N., 2008. Near-atomic resolution using electron cryomicroscopy and singleparticle reconstruction. Proceedings of the National Academy of Sciences 105 (6), $1867-1872$.

Ziv, J., Zakai, M., 1969. Some lower bounds on signal parameter estimation. IEEE Transactions on Information Theory 15 (3), 386-391. 